\title{
SOME GENERAL RESULTS ON RANDOM WALKS, WITH GENETIC APPLICATIONS
}

\author{
P. A. P. MORAN \\ (received 11 June 1962)
}

\section{Introduction}

Consider a random Markovian process in which the state of the system is defined by a random variable which can take the finite set of values $i=0,1, \cdots, N$, and which is such that transitions can only occur from any state $i$ to the two nearest states $i \pm 1$. This restriction brings about an essential simplification of the theory for the basic reason that in order for the system to move from state $i$ to state $j$ ( $i<j$ say) it must first move to $i+1$, then $i+2$ and so on until it reaches $j$. From this it follows that the first passage distribution from $i$ to $j$ is the convolution of the first passage distributions from $i$ to $i+1, i+1$ to $i+2, \cdots, j-1$ to $j$ each of which is comparatively easy to find.

In this paper we exemplify the resulting simplification in two ways. In the first we consider a continuous time process and obtain explicit representations of the Laplace transforms, with respect to time, of the first passage distributions and the transition probability densities. In the second we consider a process with one absorption state and obtain an explicit expression for the mean time of absorption. Under certain symmetry assumptions this can be generalised to the case of two absorption states and is then used to obtain the mean time required for a genetic population to become homozygous when the heterozygote is selectively advantageous over equally fit homozygotes.

\section{The continuous-time random walk}

Suppose that time is continuous and that the probabilities of moving from a state $i$ to $i-1$ and $i+1$ in the time interval $(t, t+d t)$ are equal to $\mu_{i} d t+0(d t)$ and $\lambda_{i} d t+0(d t)$ respectively. We take $\lambda_{i}>0, \mu_{i}>0$ except that $\mu_{0}=\lambda_{N}=0$. Then starting from any initial state $k$ the probabilities, $p_{i k}(t)$, of being in the state $i$ at time $t$ satisfy the differential equations:

$$
p_{0 k}^{\prime}(t)=-\lambda_{0} p_{0 k}(t)+\mu_{1} p_{1 k}(t) \text {. }
$$

$$
\begin{aligned}
& p_{i k}^{\prime}(t)=\lambda_{t-1} p_{i-1, k}(t)-\left(\lambda_{t}+\mu_{i}\right) p_{i k}(t)+\mu_{i+1} p_{i+1, k}(t) \quad(i=1, \cdots, N-1), \\
& p_{N k}^{\prime}(t)=\lambda_{N-1} p_{N-1, k}(t)-\mu_{N} p_{N, k}(t) .
\end{aligned}
$$


The resulting process is usually described as a finite Birth and Death Process with birth coefficients $\lambda_{i}$ and death coefficients $\mu_{i}$. In general the solutions of these equations is of the form

$$
p_{i k}(t)=\sum_{s=0}^{N} a_{i s} e^{\theta, t}
$$

where the $a_{i s}$ are constants chosen to fit the initial conditions, and the $\theta$, are the roots of the matrix

(3)

$$
\left[\begin{array}{ccccc}
-\left(\lambda_{0}+\theta\right) & \mu_{1} & & & \\
\lambda_{0} & -\left(\lambda_{1}+\mu_{1}+\theta\right) & \mu_{2} & & \\
& \lambda_{1} & -\left(\lambda_{2}+\mu_{2}+\theta\right) & & \\
& & \cdots & & \\
& & & \cdots & \\
& & & & \mu_{N} \\
& & & & -\left(\mu_{N}+\theta\right)
\end{array}\right]
$$

whose determinant we write as $M(\theta)$. The roots of (3) are known to be all real and distinct but it can be quickly verified that even for very simple definition of $\lambda_{i}$ and $\mu_{i}$ as functions of $i$, the $\theta_{\mathrm{s}}$ are usually very complicated and difficult to evaluate.

Karlin and McGregor $([6],[7])$ have represented the transition probabilities of Birth and Death process as the Laplace-Stieltjes transforms of products of polynomials, the integrals being taken with respect to discrete distribution functions whose points of increase are the roots of $M(\theta)$. The use of this representation involves finding these roots and it is convenient to put off doing this as long as possible. Here we give a different representation by showing how the Laplace transforms, with respect to time, of the $p_{i k}(t)$ can be represented as the ratıos of polynomials satisfying recurrence relations which are necessarily very similar to those of Karlin and McGregor.

Write

$$
P_{i k}(\theta)=\int_{0}^{\infty} e^{-\theta t} p_{i k}(t) d t,
$$

which exists for all $\theta>0$. We then have

$$
\int_{0}^{\infty} e^{-\theta t} p_{i k}^{\prime}(t)=\theta P_{i k}(\theta)-\delta_{i k},
$$

where $\delta_{i k}$ is Kronecker's $\delta$. The set of equations ( 1 ) becomes

$$
\begin{aligned}
& -\delta_{0 k}=-\left(\lambda_{0}+\theta\right) P_{0 k}(\theta)+\mu_{1} P_{1 k}(\theta), \\
& -\delta_{1 k}=\lambda_{0} P_{0 k}(\theta)-\left(\lambda_{1}+\mu_{1}+\theta\right) P_{1 k}(\theta)+\mu_{2} P_{2 k}(\theta), \\
& \cdots \\
& -\delta_{N k}=\lambda_{N-1} P_{N-1, k}(\theta)-\left(\mu_{N}+\theta\right) P_{N k}(\theta) .
\end{aligned}
$$


The matrix of coefficients on the right hand side of $(6)$ is given by (3) and thus whenever $M(\theta) \neq 0$ we can solve (6) in the form

$$
P_{i k}(\theta)=M_{i k}(\theta) M(\theta)^{-1},
$$

where $M_{i k}(\theta)$ is a matrix obtained by replacing the $(i+1)$ th column in (3) by a column consisting of zeros except for -1 in the $(k+1)$ th row. We can now find $M_{i k}(\theta)$ in a simpler form. Write $L_{i}(\theta)$ for the determinant of the matrix of the first $i+1$ rows and columns of (3), and $N_{1}(\theta)$ for the determinant of the last $N-i+1$ rows and columns. Then $L_{N}(\theta)=N_{0}(\theta)=M(\theta)$, and $L_{0}(\theta)=-\left(\lambda_{0}+\theta\right), N_{N}(\theta)=-\left(\mu_{N}+\theta\right)$. It is convenient to make the convention that $L_{-1}(\theta)=N_{N+1}(\theta)=1$.

When $i=k$ examination of $M_{i k}(\theta)$ shows that expansion by the $(i+1)$ th column gives

$$
M_{i k}(\theta)=-L_{i-1}(\theta) N_{i+1}(\theta) .
$$

By inspection of the determinant we also see that if $i>k$,

$$
M_{i k}(\theta)=(-1)^{i-k+1} \lambda_{k} \lambda_{k+1} \cdots \lambda_{i-1} L_{k-1}(\theta) N_{i+1}(\theta),
$$

and if $i<k$,

$$
M_{i k}(\theta)=(-1)^{k-i+1} \mu_{i+1} \mu_{i+2} \cdots \mu_{k} L_{i-1}(\theta) N_{k+1}(\theta) .
$$

Hence the problem of finding the Laplace transforms of the transition probabilities is reduced to that of evaluating the determinants $L_{i}(\theta)$ and $N_{i}(\theta)$ for all $i$.

Inspection of (3) shows that

$$
L_{i}(\theta)=-\left(\lambda_{i}+\mu_{i}+\theta\right) L_{i-1}(\theta)-\lambda_{i-1} \mu_{i} L_{i-2}(\theta)
$$

for $i=1, \cdots, N$, using the above convention about $L_{-1}(\theta)$, and similarly

$$
N_{i}(\theta)=-\left(\lambda_{i}+\mu_{i}+\theta\right) N_{i+1}(\theta)-\lambda_{i} \mu_{i+1} N_{i+2}(\theta) .
$$

It is useful to relate these results to first passage time distributions. Write $q_{i k}(t) d t$ for the probability that a first passage to state $i$ occurs in the interval $(t, t+d t)$ when the system was in state $k$ at time $t=0$, and define the Laplace transform by

$$
Q_{i k}(\theta)=\int_{0}^{\infty} e^{-\theta t} q_{i k}(t) d t .
$$

Similarly let $s_{k}(t) d t$ be the probability that the system remains in the state $k$ from $t=0$ until $t>0$ and then leaves $k$ in the interval $(t, t+d t)$. Clearly

$$
s_{i}(t) d t=\left(\lambda_{i}+\mu_{i}\right) e^{-\left(\lambda_{i}+\mu_{i}\right) t} d t
$$

and the Laplace transform is 


$$
S_{i}(0)=v_{i}\left(v_{i}+\theta\right)^{-1},
$$

where $\nu_{i}=\lambda_{i}+\mu_{i}$.

Because of the linear arrangement of the states and the fact that nearest neighbour transitions only are permitted we can find the $M_{i k}(\theta)$ in terms of the $Q_{i k}(\theta)$. Consider first $M_{i i}(\theta)$.

$p_{i i}(t)$ is the probability that the system is in state $i$ at time $t$ if it was also in state $i$ at $t=0$. This could have happened in three different ways.

In the first there might have been no change in the interval $(0, t)$. This has probability $e^{-v_{i} i}$. In the second the system stays in $i$ until a time $u(<t)$, passes to $i-1$ in $(u, u+d u)$, passes back to $i$ for the first time after $u$ in $(v, v+d v)$, where $u<v<t$, and then whatever happens after $v$, is in $i$ at time $t$. The probability of this sequence is

$$
\mu_{i} e^{-v i u} q_{i, i-1}(v-u) p_{i i}(t-v) d u d v .
$$

The third possibility is similar but the system moves to $i+1$ in $(u, u+d u)$ and back to $i$ for the first time in $(v, v+d v)$. Adding tinese possibilities we have

$$
\begin{aligned}
p_{i i}(t)= & e^{-v_{i} t}+\int_{0}^{t} \int_{0}^{v} e^{-v_{i} u} \mu_{i} q_{i, i-1}(v-u) p_{i i}(t-v) d u d v \\
& +\int_{0}^{t} \int_{0}^{0} e^{-v_{i} u} \lambda_{i} q_{i, i+1}(v-u) p_{i i}(t-v) d u d v .
\end{aligned}
$$

Taking Laplace transforms we have

$$
\begin{aligned}
P_{i i}(\theta)= & \left(v_{i}+\theta\right)^{-1}+\mu_{i}\left(v_{i}+\theta\right)^{-1} Q_{i, i-1}(\theta) P_{i i}(\theta) \\
& +\lambda_{i}\left(v_{i}+\theta\right)^{-1} Q_{i, i+1}(\theta) P_{i i}(\theta)
\end{aligned}
$$

and hence

$$
P_{i i}(\theta)=\left\{\nu_{i}+\theta-\mu_{i} Q_{i, i-1}(\theta)-\lambda_{i} Q_{i, i+1}(\theta)\right\}^{-1} .
$$

If $k<i$, it is also clear that

$$
Q_{i k}(\theta)=Q_{k+1, k}(\theta) Q_{k+2, k+1}(\theta) \cdots Q_{i, i-1}(\theta),
$$

and

$$
P_{i k}(\theta)=Q_{i k}(\theta) P_{i i}(\theta) \text {. }
$$

Next we must find the $Q$ 's. Consider $Q_{i+1, i}(\theta)$. A first passage from $i$ at $t=0$ to $i+1$ at $t$ can occur in two ways. In the first there is no change between $t=0$ and $t-d t$, but during $(t-d t, t)$ there is a jump from $i$ to $i+1$. This has probability

$$
\lambda_{i} e^{-y_{i} t} d t
$$

In the second there is no change between 0 and $u(0<u<t)$, a jump to $i-1$ in $(u, u+d u)$, a first passage from $i-1$ to $i$ in $(v, v+d v)$ 
$(0<u<v<t)$, and a first passage from $i$ to $i+1$ in $(t-d t, t)$. Integrating over the values of $u$ and $v$, this has probability

$$
\int_{0}^{t} \int_{0}^{v} e^{-v_{i} u} \mu_{i} q_{i, i-1}(v-u) q_{i+1, i}(t-v) d u d v d t .
$$

Taking Laplace transforms we obtain

$$
Q_{i+1, i}(\theta)=\lambda_{i}\left(v_{i}+\theta\right)^{-1}+\mu_{i}\left(v_{i}+\theta\right)^{-1} Q_{i, i-1}(\theta) Q_{i+1, i}(\theta),
$$

and hence

$$
Q_{i+1, i}(\theta)=\lambda_{i}\left\{v_{i}+\theta-\mu_{i} Q_{i, i-1}(\theta)\right\}^{-1},
$$

which provides us with a recurrence relation for the $Q_{i+1, i}(\theta)$ from which they can be found successively starting from

$$
Q_{1,0}(\theta)=\lambda_{0}\left(\lambda_{0}+\theta\right)^{-1} \text {. }
$$

Similarly we have

$$
Q_{i-1, i}(\theta)=\mu_{i}\left\{\nu_{i}+\theta-\lambda_{i} Q_{i, i+1}(\theta)\right\}^{-1},
$$

$$
Q_{N-1, N}(\theta)=\mu_{N}\left\{\mu_{N}+\theta\right\}^{-1} .
$$

We now show that

$$
Q_{i+1, i}(\theta)=-\lambda_{i} L_{i-1}(\theta) L_{i}(\theta)^{-1},
$$

and

$$
Q_{i-1, i}(\theta)=-\mu_{i} N_{i+1}(\theta) N_{i}(\theta)^{-1} .
$$

Equation (22) is clearly true for $i=0$ from (19) and the known values of $L_{-1}(\theta)$ and $L_{0}(\theta)$. Supposing that (22) is satisfied for all values of $i$ up to $i=s$, substituting in (18) and using the recurrence relation (11) we see that (22) is true for $i=s+1$, and therefore for $i=0,1, \cdots N$. (23) may be similarly verified.

Substituting in (16) and (17) we find for $k<i$,

$$
\begin{aligned}
& P_{i k}(\theta)=\frac{(-i)^{i-k} \lambda_{k} \cdots \lambda_{i-1} L_{k-1}(\theta) N_{i+1}(\theta)}{\left\{\left(\nu_{i}+\theta\right) L_{i-1}(\theta) N_{i+1}(\theta)-\mu_{i} \lambda_{i-1} L_{i-2}(\theta) N_{i+1}(\theta)-\lambda_{i} \mu_{i+1} L_{i-1}(\theta) N_{i+2}(\theta)\right\}},
\end{aligned}
$$

which is otherwise obvious by expanding (7).

The advantage of this method of procedure is that in any particular case we can obtain $L_{i}(\theta)$ and $N_{N-i}(\theta)$ explicitly for small values of $i$ and knowing the recurrence relation we can try to guess the resulting polynomials. A systematic investigation of all the simplest functions $\lambda_{i}, \mu_{i}$ would be desirable. Here we draw attention to a few known cases. 


\section{Special cases}

If either all the $\lambda_{i}$, or all the $\mu_{i}$, are zero, the roots of (3) are obvious and it is not in principle difficult to write down an explicit solution. In particular if all $\mu_{i}=0$ and the $\lambda_{i}$ are constant the equations are easy to solve. If $\mu_{i}=0$ and $\lambda_{i}=\lambda i$ we have the well-known linear birth process. If $\mu_{i}=0$ and $\lambda_{i}$ is a quadratic function of $i$ we have a quadratic birth process considered by John [5].

If $\lambda_{i}$ and $\mu_{i}$ are both non-zero constants the recurrence relations can be solved explicitly and inversion of the Laplace transforms results in modified Bessel functions of the first kind (Heathcote and Moyal [3]).

Not many other explicit solutions are known. When $N$ is infinite and $\lambda_{i}=\lambda i, \mu_{i}=\lambda i$ we have the well known simple birth and death process first solved by Palm (see Kendall [9]). It is usually more realistic in practice to put an upper bound to the size of the population, thus making the methods of the present paper applicable, and for biological populations the natural approach is to construct an analogue of the deterministic logistic process in the manner described by Kendall. We suppose that the population is confined between two limits $N_{1}, N_{2}$ so that $0 \leqq N_{1} \leqq i \leqq N_{2}$, and that the birth and death rates per individual are linear functions of $i$;

$$
\begin{aligned}
& \lambda_{i}=\lambda i\left(N_{2}-i\right), \\
& \mu_{i}=\mu i\left(i-N_{1}\right) .
\end{aligned}
$$

This makes the death rate per individual increase linearly with $i$, and the birth rate per individual decrease linearly. In order to get simpler equations Prendiville [14] suggested that (25) and (26) be modified to

$$
\begin{aligned}
& \lambda_{i}=\lambda\left(N_{2}-i\right), \\
& \mu_{i}=\mu\left(i-N_{1}\right),
\end{aligned}
$$

thus making the birth and death rates per individual proportional to $N_{2} i^{-1}-1$ and $1-N_{1} i^{-1}$ respectively, which vary in the right direction. He did not, however, publish his analysis and the first published solution was given by Takashima [15].

The present theory makes the reason for the simplicity of the solution clearer. Shifting the origin of definition of $i$ to $N_{1}$ we can write (27) and (28) in the form

$$
\begin{aligned}
& \lambda_{i}=\lambda(N-i), \\
& \mu_{i}=\mu i,
\end{aligned}
$$

and the roots of the determinant $M(\theta)$ come out simply by using the factorisation of the determinant 


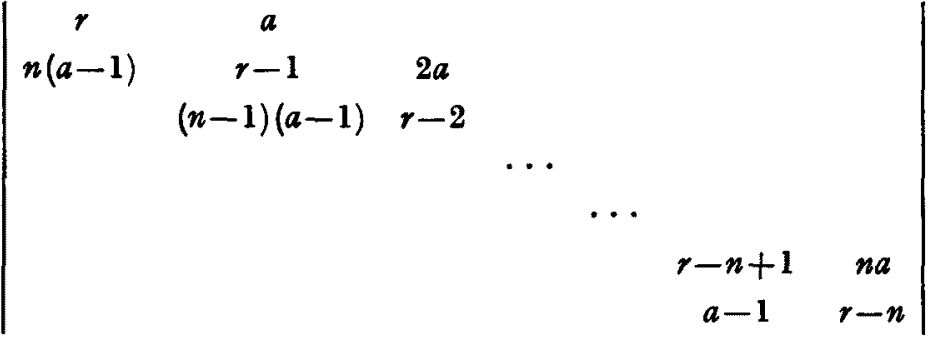

$$
\begin{aligned}
& =\prod_{S=0}^{n}(r+n a-n-s(2 a-1)),
\end{aligned}
$$

which is given by Painvin [13] and described in Muir [12] p. 432. This can be easily reduced to the above case.

Another process in which the roots can be found explicitly is the continuous time analogue of a genetic model due to the author which has been discussed in detail by Karlin and McGregor [8].

\section{First passage times when $t$ is discrete}

It is clear that the above theory can be developed in an exactly analogous manner for discrete time processes, generating functions replacing the Laplace transforms. We do not do this here but consider only a closely related problem of determining the mean first passage time to an absorbing state in a general random walk described by a Markov chain with discrete time. This was developed for the purpose of obtaining a partial answer to an unsolved problem in population genetics, that of finding the time required for a population to become homozygous when heterozygotes are selectively advantageous and there is no mutation.

Suppose then that we have a Markov chain with $N+1$ states, $i=0,1, \cdots N$ such that the probabilities of a jump from $i$ to $i-1, i, i+1$ are $q_{i}, r_{i}, p_{i}$ respectively, where $q_{i}+r_{i}+p_{i}=1$, and $q_{0}=p_{N}=0$. If $p_{0}=q_{N}=0$ the states $i=0, N$ are absorbing, and we suppose there are no other absorbing states. Let $T_{i}$ be the mean time, measured in units of one jump, for the system to reach an absorbing state starting from the state $i$. Put $T_{0}=T_{N}=0$, and suppose all states are ultimately accessible from the states $i=1, \cdots N-1$. Then the $T_{i}$ are finite and

$$
T_{i}=1+q_{i} T_{i-1}+r_{i} T_{i}+p_{i} T_{i+1},
$$

for $i=1, \cdots N-1$. These give $N-1$ equations for $N-1$ unknowns but in general are not easily soluble. If, however, there is only one absorbing state an explicit solution is simple. Suppose this absorbing state is $i=M$ so that $p_{0}>0$. Let $t_{i}$ be the mean first passage time from $i$ to $i+1$. Then 


$$
T_{i}=t_{i}+t_{i+1}+\cdots+t_{N-1} .
$$

If the system is in state $i$ it will remain there for a period $s_{i}$ and then move either to $i-1$ or to $i+1$, where $s_{i}$ clearly has a geometric distribution,

Thus

$$
\operatorname{prob}\left(s_{i}=n\right)=r_{i}^{n-1}\left(1-r_{i}\right), \quad n=1,2, \cdots
$$

$$
E\left(s_{i}\right)=\left(1-r_{i}\right)^{-1} .
$$

If the system moves to $i+1, s_{i}$ will be the first passage time. If it moves to $i-1$ the expected remaining time to reach $i+1$ will be $t_{i-1}+t_{i}$.

Thus

so that

$$
t_{i}=\left(1-r_{i}\right)^{-1}+q_{i}\left(t_{i-1}+t_{i}\right)\left(1-r_{i}\right)^{-1}
$$

$$
p_{i} t_{i}=1+q_{i} t_{i-1} .
$$

It is also possible to obtain a recurrence formula for the variance of the first passage time from $t$ to $t+1$ but this is more complicated.

Since $t_{0}=p_{0}^{-1}$ we can find $t_{1}, t_{2}, \cdots$ in succession, thus obtaining

$$
\begin{aligned}
& t_{1}=\frac{1}{p_{1}}+\frac{q_{1}}{p_{0} p_{1}}, \\
& t_{2}=\frac{1}{p_{2}}+\frac{q_{2}}{p_{1} p_{2}}+\frac{q_{1} q_{2}}{p_{0} p_{1} p_{2}},
\end{aligned}
$$

and in general

$$
\begin{aligned}
t_{i} & =\frac{1}{p_{i}}+\frac{q_{i}}{p_{i-1} p_{i}}+\cdots+\frac{q_{1} \cdots q_{i}}{p_{0} p_{1} \cdots p_{i}} \\
& =\sum_{k=0}^{k=i} \frac{q_{i+1} q_{i} \cdots q_{i-k+1}}{p_{i+1} p_{i} p_{i-1} \cdots p_{i-k}} .
\end{aligned}
$$

From (35) $T_{1}$ can be found by summation. These results can be found in Harris [2] and Chung (see references in the latter's book [1]), and also Hodges and Rosenblatt [4]. Notice that similar methods can be used with continuous time models.

The above theory depends essentially on the assumption that there is only one absorption state. However, we can still deal with the case where both $i=0$ and $i=N$ are absorbing states if we have the symmetry condition $q_{i}=p_{N-i}$. To do this we identify the states $i$ and $N-i$ which is now permissible. If $N$ is even and equal to $2 M$ we get a Markov chain with $M+1$ states $E_{0}, E_{1}, \cdots, E_{M}$ such that $E_{0}$ is not an absorbing state but $E_{M}$ is. 


\section{An application to genetics}

We now apply this result to discuss the behaviour of some genetic populations. The majority of such populations consist of diploid individuals but the mathematics is then so complicated that for the most part mathematical geneticists have been content to study models in which the individuals are haploid and the effects of selection are of such a kind as to provide a good imitation of the diploid case.

One model of such a population is as follows (Moran [10] [11]). Suppose the population consists of $N=2 M$ haploid individuals which are genetically either $A$ or $a$. The number of $A$ individuals is $i$ and defines the state of the system where $i=0,1, \cdots, N$. At successive unit intervals of time a haploid individual, chosen at random, dies and is replaced by a new individual which is $A$ or $a$ with probabilities equal to the relative proportion of these in the population before the death occurred. If the system was in the state $i$, it can only move to the states $i-1, i$, and $i+1$ at each step, and does so with probabilities,

$$
\begin{aligned}
& p_{i-1, i}=i(N-i) N^{-2}, \\
& p_{i, i}=\left\{i^{2}+(N-i)^{2}\right\} N^{-2}, \\
& p_{i+1, i}=i(N-i) N^{-2} .
\end{aligned}
$$

The behaviour of this process has been very thoroughly studied and in particular Watterson [16] showed that if it starts from the state $j$, the expected number of steps to one of the absorbing states $0, N$, is given by

$$
\begin{aligned}
E\left(T_{j}\right)= & N(N-j)\left\{(N-1)^{-1}+\cdots+(N-j)^{-1}\right\} \\
& +N j\left\{(N-1)^{-1}+\cdots+(j-1)^{-1}\right\} .
\end{aligned}
$$

This can be obtained by the above methods as we shall see, and so also can Watterson's similar formula for $\operatorname{Var}\left(T_{3}\right)$.

One of the main problems in the study of genetic models of this kind is to determine how fast real genetic populations become homozygous as the result of random assortment of genes. In the haploid models this corresponds to the states $j=0, N$ and we often use the expression "rate of progress to homozygosity" for such models in spite of the fact that there are no zygotes, the individuals being haploid. Thus what we are really concerned with is the rate of absorption into the states $j=0, N$ and in so far as the haploid model is an adequate description of the real diploid population, this provides an answer to the problem.

The probability that $j \neq 0, N$ will be asymptotically equal to $C \lambda^{t}$, where $C$ is a constant, $\lambda$ is the largest non-unit root of the matrix of transition probabilities, and $t$ is the number of birth-death events. Much research has been devoted to the calculation of $C$ and $\lambda$ for varying genetic 
assumption but hardly any progress has been made when there is any kind of selection. In particular the most interesting case arises when, in populations of diploids, the heterozygote $A a$ has a selective advantage over the two homozygotes $A A$ and $a a$. This leads to the simplest example of a "selectively balanced polymorphism", and it is of great practical interest to determine how large such selective forces must be to keep a natural population polymorphic for a given number of generations.

In such circumstances $\lambda$ has not been found and would be extremely difficult to find theoretically. However, a different approach can be made by calculating not the probability that the process is heterozygous as a function of $t$, but simply the mean time necessary to become homozygous. We shall show that the mathematics is then not difficult provided the two homozygotes, $A A$ and $a a$, have equal selective value.

In a haploid model, diploid selection has no meaning. However, a satisfactory model could be constructed by modifying the transition probabilities in the haploid model in such a way that the change in gene frequency from $t$ to $t+1$ has the mean and variance it should have in a diploid model.

Consider first what happens in an infinitely large population with diploid individuals which are $A A, A a$, or $a a$. Let the frequency of the $A$ gene be $p$, and assume random mating so that the frequencies of the $A A, A a$, and $a a$ individuals are $p^{2}, 2 p q$, and $q^{2}(q=1-p)$. Suppose that $A A$ and $a a$ have the same selective value unity, and that the heterozygote $A a$ has the relative selective value $1+s$ where $s>0$. It is then well known and easy to show that the expected change in gene frequency from one generation to the next is approximately

$$
\Delta p=-s p q(p-q)
$$

the approximation being satisfactory when $s$ is small compared with unity.

Since $i N^{-1}$ is the relative gene frequency in the haploid model, and since one generation corresponds to $N$ birth-death events, a suitable model is given by

$$
\begin{aligned}
& p_{i+1, i}=p_{i}=i(N-i) N^{-2}\left\{1-\frac{1}{2} s N^{-1}(2 i-N)\right\} \\
& p_{i, t}=r_{i}=\left\{i^{2}+(N-i)^{2}\right\} N^{-2} \\
& p_{i-1, i}=q_{i}=i(N-i) N^{-2}\left\{1+\frac{1}{2} s N^{-1}(2 i-N)\right\} .
\end{aligned}
$$

We can now find the mean time of absorption. Suppose $N$ is even and equal to $2 M$. Since $p_{i}=q_{N-1}$ we can replace the system by a Markov chain with $M+1$ states $E_{k}, k=0,1, \cdots, M$, for which

$$
\begin{array}{llrl}
p_{k+1, k}=p_{k}=\left(4 M^{2}\right)^{-1}\left(M^{2}-k^{2}\right)\left\{1-\frac{1}{2} s k M^{-1}\right\}, & & (k>0), \\
p_{k, k}=r_{k}=\left(2 M^{2}\right)^{-1}\left(M^{2}+k^{2}\right), & & \\
p_{k-1, k}=q_{k}=\left(4 M^{2}\right)^{-1}\left(M^{2}-k^{2}\right)\left\{1+\frac{1}{2} s k M^{-1}\right\}, & & (k>0) .
\end{array}
$$


We substitute from these in (32) and (35) and obtain

$$
E\left(T_{i}\right)=\sum_{j=i}^{M-1} \sum_{k=0}^{j} A_{j k}
$$

where

$$
\begin{aligned}
A_{j k} & =\frac{4 M^{2}(N+s j) \cdots(N+s(j-k+1))}{\left\{M^{2}-(j-k)^{2}\right\}\left\{1-\frac{1}{2} s(j-k) M^{-1}\right\}(N-s j) \cdots(N-s(j-k+1))} \\
& =\frac{4 M^{2} \Gamma\left(N s^{-1}+j+1\right) \Gamma\left(N s^{-1}-j\right)}{\left\{M^{2}-(j-k)^{2}\right\}\left\{1-\frac{1}{2} s(j-k) M^{-1}\right\} \Gamma\left(N s^{-1}+j-k+1\right) \Gamma\left(N s^{-1}+k-j\right)} \cdot
\end{aligned}
$$

We assume that $s$ is of the same order as $M^{-1}$ and we put $s=\sigma M^{-1}$ where $\sigma$ is to be kept fixed as $M$ gets large. Consider the expression

$$
\Gamma(x+a) \Gamma(x+b)^{-1}
$$

where $a$ and $b$ are kept fixed and $x$ increases indefinitely. Using Stirling's formula this is asymptotically equal to

$$
\begin{array}{r}
x^{a-b} \exp \left\{b-a+\left(x+a-\frac{1}{2}\right) \log \left(1+a x^{-1}\right)\right. \\
\left.-\left(x+b-\frac{1}{2}\right) \log \left(1+b x^{-1}\right)\right\} .
\end{array}
$$

Expanding the logarithm and taking the largest term we get

$$
x^{a-b} \exp \left\{\frac{1}{2}\left(a^{2}-b^{2}\right) x^{-1}-\frac{1}{2}(a-b) x^{-1}\right\} .
$$

Using this result in (40) we find that $A_{j k}$ is asymptotically equal to

$$
4 M^{2}\left\{M^{2}-(j-k)^{2}\right\}^{-1} \exp \frac{1}{2} \sigma M^{-2} k(2 j-k+1) \text {. }
$$

We can therefore approximate to the double sum in (39) by the integral

$$
4 M^{2} \int_{x}^{1} \int_{0}^{y}\left\{1-(y-z)^{2}\right\}^{-1} \exp \frac{1}{2} \sigma z(2 y-z) d z d y,
$$

where $x=i M^{-1}$, $i$ being the initial state. Putting $u=y-z$ this becomes

$$
4 M^{2} \int_{x}^{1} \int_{0}^{v}\left(1-u^{2}\right)^{-1} \exp \frac{1}{2} \sigma\left(y^{2}-u^{2}\right) d u d y .
$$

In the particular case $x=0$ so that the process starts with the numbers of $A, a$ genes equal, this is easily transformed into

$$
E\left(T_{i}\right)=4 M^{2} \int_{0}^{1}\left(1-u^{2}\right)^{-1} \exp -\frac{1}{2} \sigma u^{2} \int_{u}^{1} \exp \frac{1}{2} \sigma y^{2} d y d u,
$$

which can be fairly easily evaluated in particular cases by numerical integration. When $\sigma=0,(41)$ can be verified to be asymptotically to equal to (37) which can also be derived directly from (39). 


\section{References}

[1] Chung, K. L., Markov Chains with stationary transition probabilities. Grundlehren der Mathematischen Wissenschaften. Band 104. Springer (1960).

[2] Harris, T. E., First passage and recurrence distributions. Trans. Amer. Math. Soc. 73, (1952) $471-486$.

[3] Heathcote, C. R. and Moyal, J. E., The random walk (in continuous time) and its application to the theory of queues. Biometrika 46, (1959) 400-411.

[4] Hodges, J. L. and Rosenblatt, M., Recurrence time moments in random walks. Pacific J. Math. 3, (1953) 127-136.

[6] John, P. W. M., The quadratic birth process (Abstract). Ann. Math. Statistics 27, (1956) 865.

[6] Karlin, S. and McGregor, J. L., The differential equations of birth-and-death processes and the Stieltjes moment problem. Trans. Amer. Math. Soc. 85, (1957) 489-546.

[7] Karlin, S. and McGregor, J. L., The classification of birth-and-death processes. Trans. Amer. Math. Soc. 86, (1957) 366-400.

[8] Karlin, S. and McGregor, J. L., On a genetics model of Moran. Proc. Cam. Phil. Soc. 58, (1962).

[9] Kendall, D. G., Stochastic processes and population growth. Jour. Roy. Statist. Soc. B. 11, (1949) 230-264.

[10] Moran, P. A. P., Random processes in genetics. Proc. Cam. Phil. Soc. 54, (1958) 60-71.

[11] Moran, P. A. P., The statistical processes of evolutionary theory. Oxford University Press. 1962.

[12] Muir, I., History of the Theory of Determinants. Vol. 3, (1911) p. 432.

[13] Painvin, L., Sur un certain système d'équations linéaires. Jour. de Math. (de Liouville) (2) iii, (1858) 41-46.

[14] Prendiville, B. J., Discussion of a paper by D. G. Kendall. Jour. Roy. Stat. Soc. B. 11 , (1949) 273.

[15] Takashima, M., Note on evolutionary processes. Bull. Math. Stat. 7, (1956) 18-24.

[16] Watterson, G. A., Markov chains with absorbing states: a genetic example. Ann. Math. Stat. 32 (1961) $716-729$.

Institute of Advanced Studies, The Australian National University, Canberra 